\title{
Some Thoughts on Development Evaluation Processes*
}

\section{Ole Winckler Andersen}

\begin{abstract}
A$ lively methodological debate together with organisational adjustments has dominated the international discussion on development evaluation in the last decade. Less discussed have been the evaluation processes, from procurement of consultants to the completion of evaluations, where many donors have used the same 'system' with only minor adjustments for many years. A key question is whether this 'system' is still 'fit for purpose' in view of the increasing complexity of many development interventions. This article presents three perspectives on development evaluation processes - the 'independence perspective', the 'transaction cost perspective' and the 'political economy perspective' - and argues that in particular the 'political economy perspective' constitutes a relevant perspective in analyses of evaluation processes. It is concluded that evaluation processes should be designed to reflect the specific characteristics of the individual evaluations, but also that more analysis and experimentation are required.
\end{abstract}

\section{Introduction}

In recent years there has been a lot of discussion of evaluation methodologies ${ }^{1}$ and increasingly also of complexity in development. ${ }^{2}$ These discussions seem to be driven by an implicit or explicit acknowledgement of a need to develop and adjust the applied methods to new contexts and policy agendas. In particular, complex and adaptable programmes are becoming more prominent, but diversification is widespread and some donors seem even partly to have returned to an increased use of project assistance. New financing instruments and the fact that new actors engage in evaluation processes contribute to the challenges. These discussions and their implications for development evaluation will probably continue and become even more pronounced with the upcoming post 2015-agenda, where more comprehensive global goals are foreseen as well as increasing integration of development policies with other policy areas (United Nations 2013).

While debates on methodology, on problems with development evaluation quality, ${ }^{3}$ and on how donors organise their evaluation units have been intensive, there has been less discussion of the evaluation process itself ${ }^{4}$ and the use of evaluation consultants in particular. ${ }^{5}$ Development evaluations can be performed in different ways.
Some donors transfer the full responsibility for the evaluations to external consultants, others have internal team leaders assisted by external consultants, and a few donors have internal evaluation units, which have full responsibility for carrying out the evaluations. In spite of this variation, a feature of many development evaluations is a significant use of external evaluation consultants, who are contracted on short-term contracts based on Terms of Reference (TOR) elaborated by the donor evaluation units.

This 'system', which was established at a time when project assistance was the most common approach, has been used for many years without much discussion and adjustment, and the general tendency is that the same system and process is applied by the individual donors irrespectively of the character of the evaluation. The reason for this absent discussion is probably that the independence and use of external consultants by many are seen as being related, and that there is a fear of discussing the role of consultants and the evaluation processes as that may question the independence and credibility of development evaluations.

This article provides some thoughts on the evaluation process and argues that there is an 
urgent need to consider how the organisation of development evaluation can be adapted to the challenges of development evaluation. After this introduction, the article continues with a brief presentation of the 'market' for development evaluation followed by a discussion of different perspectives, which can be applied to the organisation of evaluation processes. It is shown that each of the presented perspectives can lead to specific recommendations on how to organise the evaluations, but also that there is a need for experimentation and further analyses.

\section{The 'market' for development evaluation}

In 2013 OECD-DAC members provided US $\$ 135$ billion in development assistance. ${ }^{6}$ According to OECD-DAC, these donors produce more than 600 evaluations, including decentralised evaluations, per year and on average use $0.5-1.0$ per cent of their development assistance on development evaluation (OECD 2010a). This implies that US $\$ 0.6-1.3$ billion is spent annually on development evaluation. To this can be added evaluation budgets of new donors, private foundations, etc. Their budgets can, however, be assumed to be relatively small compared to the OECD-DAC donors' budgets for evaluation. Most evaluation budgets are used for different purposes related to evaluation (own staff costs, preparation and dissemination of evaluations, etc.) and not only for development evaluation consultants. If it is assumed that other donors than the OECD-DAC donors use 10 per cent of what is used by the OECD-DAC donors, and that approximately 50 per cent are used on development consultants, the size of the market for development evaluation consultants is about US\$300-600 million per year. This is obviously a very rough estimate, and the assumptions can clearly be questioned, ${ }^{7}$ but the main observation is that the market has a significant size.

The consultants are usually contracted on relatively standardised short-term contracts based on TOR drafted by an evaluation unit, ${ }^{8}$ and the procedures will be similar to those used in procurement of other goods and services. This entails that the same procedures are used whether the donor will fund a construction of a road, a project evaluation or an evaluation of a complex development intervention.

The demand for development evaluations has increased significantly over the last decade and probably to an extent that the supply of development evaluation consultants has had difficulties following this growth (see e.g. DFID 2014: 54, 57). The supply of development evaluation consultants is relatively limited and entry barriers are high in particular for team leaders of development evaluations, i.e. due to procurement procedures, where significant consultant experience is often required. This stability in the supply of evaluation development consultants combined with a relatively low rotation of staff members of evaluation units implies that there will be continuous and repeated interaction between them, and both evaluation consultants and staff of evaluation units will probably consider both the short term (the present contract) and the long term (potential future collaboration), when an evaluation is carried out (Andersen and Broegaard 2012: 50). In addition, while some evaluations may be conducted without much contact between evaluation consultants and evaluation units during the evaluation processes, most evaluation processes today involve continuous and close contact. It is reasonable to assume that this contact is more pronounced for evaluations of complex interventions, where the drafting of TOR, including the design of the methodological approach, will often be a challenge due to insufficient information about the context, interventions, local actors, and also about data availability and feasibility of conducting fieldwork. Therefore evaluations of complex interventions will often require more discussion and consultation on the interpretation of TOR, on methodological issues and the practical organisation of the fieldwork. Also the strengthened focus on the use of evaluations has led to more contact and consultation during the evaluation process not only between consultants and evaluation units, but also with other stakeholders. Thus, in particular two issues characterise the market for development evaluation and distinguish it from most competitive markets: the repeated interaction between evaluation consultants and evaluation units, and their frequent contact during the individual evaluation processes.

\section{Perspectives on development evaluation processes}

As indicated, there may be different perspectives on the organisation of development evaluation processes. In the following a distinction will be made between three perspectives, which can potentially lead to different implications and 
recommendations for how to organise the evaluation process. The perspectives will only briefly be introduced.

\subsection{The 'independence perspective'}

This is the dominating perspective in discussions on the organisation of development evaluations. The key argument for using external consultants is that it ensures independent evaluations, which is considered important for ensuring both the quality of the evaluations and their credibility. Discussions in connection with this perspective have in particular focused on various institutional mechanisms, which can ensure the independence of the development evaluations. The proposed mechanisms can for instance focus on transparency in tender procedures and the position and mandate of the evaluation unit (see Picciotto 2013: 30-32). As the argument for independence presupposes the professionalism of evaluation consultants, a further element in the discussions related to this perspective has been the degree to which it can be argued that development evaluation is a profession and that professionalism is the key motivating factor for evaluation consultants in evaluation processes (Jacob and Boisvert 2010; Picciotto 2011). ${ }^{9}$

However, it has also been argued that the study of evaluation independence has been neglected (Picciotto 2013: 18), and it is notable that international evaluation norms and standards have their focus on basic principles of independence, but do not consider how the evaluation independence can be influenced by different characteristics of the development evaluations and of the evaluation processes (compare with e.g. OECD 2010b). The independence of evaluations could in particular be relevant to consider if there is frequent contact and dialogue between consultants and evaluation units throughout the evaluation processes, if it is a small relatively stable group of consultants where there is a mutual dependency between them and evaluation units due to their repeated interactions, or when the evaluation is of complex interventions, where it can be difficult to draft detailed and precise TOR, and the interpretation of the TOR is as a consequence established through negotiation during the evaluation process.

Although the perspective recommends a market approach there has been limited focus on the cost of the evaluations, and it seems often at least implicitly assumed - although apparently never systematically analysed - that the market provides an efficient solution to procurement of evaluation consultants. As mentioned above the general supply of consultants may, however, have difficulties in meeting an increasing demand, which can lead to increasing evaluation costs (see DFID 2014: 53).

\subsection{The 'transaction cost perspective'}

This perspective has been less discussed in connection with development evaluation, but would take its inspiration from transaction cost economics. ${ }^{10}$ The argument would be that the production of evaluations should be organised in a way that minimises their transaction costs with the implication that the organisation of evaluations should reflect the specific characteristics of the evaluations. In this perspective the market solution and the internal solution are considered as alternative modes of governance, and it thus leaves room for different organisational solutions. The transaction cost economics approach has developed a number of principles - for example asset specificity, frequency and uncertainty (see Williamson 1998: 36) - which can be used to consider and compare the relevance of different organisational solutions. The arguments from the transaction cost economics literature imply that standard and relatively simple evaluations (or evaluation tasks) could be out-sourced, while more complex tasks of a recurrent nature should be carried out in-house, and that the out-sourcing of complex tasks could lead to high transaction costs. These should obviously be compared to the transaction costs associated with an internal solution, but one description of the continuous dialogue and endless discussions between consultants and evaluation units during a complex evaluation in South Sudan clearly demonstrates the potentially high transaction costs, which can be encountered during a development evaluation (Barnett and Bennett 2014: 53-4).

While this perspective may provide some important insights, it seems obvious that the production of evaluations - i.e. due to the repeated interaction between consultants and evaluation units, and their contact during the evaluation processes cannot just be compared to the production of other goods and services. The characteristics of external and internal organisational solutions may therefore differ substantially from other areas of production, and the delimitation and 
measurement of the transaction costs would entail particular challenges. Moreover, the incentives and motivations are complex and could, for instance, differ among different groups of stakeholders or be linked to different degrees of professionalism among the development evaluation consultants, and it is not convincing that the rather simple behavioural assumptions of the transaction cost economics approach capture these various incentives, or that transaction cost minimisation should guide how evaluation processes are organised.

\subsection{The 'political economy perspective'}

This perspective, which can be considered as an extension of the transaction cost perspective, provides a more nuanced framework for analysing the interaction between evaluation consultants and evaluation units during the evaluation process. ${ }^{11}$ Like the transaction cost perspective this perspective has attracted less attention in discussions of evaluation processes. The perspective will have its focus on the interactions between the different actors, information problems and asymmetric information, complex preference structures and incentives, and on actors' behaviour and strategies in these complex settings and in view of the often repeated character of the interactions. The framework can also include other actors (the general public, politicians, donor agencies, etc.) and be used as a framework for analyses of different types of influence, including power (see e.g. Furubo and Vestman 2011). The perspective, which seeks to move behind descriptive or normative discussions, thus recognises that the interactions between consultants and evaluation units have a very complex nature, and that analyses of the evaluation processes as a consequence will have to be based on an understanding of these interactions. As the number of analyses of the evaluation processes is very limited, an implication would be that recommendations about organisational solutions can first be made after more information has been collected and analysed. The perspective would, however, probably lead to similar conclusions as the transaction cost perspective in which it was argued that organisational solutions should reflect the characteristics of the evaluation, but with the difference that these 'characteristics' in this third perspective will comprise a larger number of different elements - and not only transaction costs. Both the transaction cost and the political economy perspective would therefore argue that the contract procedures and the evaluation process will influence the incentives of the various actors, including the evaluation consultants, and therefore must be designed taking this into consideration. This contrasts with the present practice, where most donor evaluation units tend to use the same evaluation process for all evaluations without contemplating the wide variation in the characteristics of the evaluations, including the increasing complexity of many evaluations.

\section{Need for empirical analyses and experimentation}

Although evaluation processes is often the preferred discussion topic, when evaluation consultants and staff from donor evaluation units meet, there is insufficient systematic empirical knowledge about these processes and the strategies different actors apply. ${ }^{12}$ Instead, perceptions of the evaluation processes seem to be based on rather simple - and untested - assumptions about the behaviour of evaluation consultants and evaluation units, and without consideration for the changing landscape of development evaluation and the associated challenges. Due to the complexity of many evaluation processes, further analytical work should therefore be supplemented by collection of available experiences and monitored experimentation with potential organisational solutions. Methodologically, monitoring and learning from such experiences will be a challenge due to the significant variation in contexts, actors and evaluations, as well as the vested interests of the various actors, and will require careful planning. In the following, three, not mutually exclusive, areas for further analyses and experimentation will be briefly presented.

\subsection{Empirical analyses of the behaviour of consultants and evaluation units}

While all who have been involved in development evaluations can give many examples of highly professional consultants with high ethical standards in their work, the question is how in practice this professionalism is balanced against considerations for, for example, company profits and future contracts. Both evaluation consultants and evaluation units may therefore be guided by a complex set of interests and strategies, and they may prioritise these interests and strategies differently in the individual evaluations.

As mentioned above these discussions have been seen as closely related to discussions of independence. If professionalism is the dominant 
motivating factor for development evaluation consultants, this could lead to a demand for independence and flexibility in the evaluation process; and a market solution could be the preferred organisational solution. On the other hand, if professionalism is not dominating or if staff of evaluation units possess a similar professional approach, the argument that external solutions are required to ensure independence would be less convincing. A particular issue is which factors influence the strategies of the evaluation consultants, and whether their affiliation (consultancy company, freelance, research institution, university, etc.) plays a significant role.

In addition to empirical analyses of the behaviour of evaluation consultants, analyses of the behaviour of evaluation units on the evaluation market and in the evaluation process would be warranted. Evaluation units are often either presented as neutral evaluation commissioners or it is argued that internal evaluation units are not autonomous in spite of various safeguards. These descriptions are obviously very general and do not in any detail reflect the behaviour of evaluation units and their staff or the complexity of the evaluation processes (see Andersen and Broegaard 2012). For instance, reasonable assumptions will be that the institutional set-up of the units will influence the behaviour of the staff, and that staff of evaluation units will also be guided by some degree of professionalism. A particular issue in the evaluation process is the strategies evaluation units apply if the work of the evaluation consultants is not found to meet the expected quality (see e.g. ITAD 2014: 73).

The above raises a number of questions on the behaviour of not only evaluation consultants, but also evaluation units and their staff, which can only be answered empirically. There is therefore a need for further empirical work on incentives and strategies of the different actors in different contexts, including in connection with different types of evaluations, and as indicated the political economy perspective will provide an adequate analytical framework for such analyses.

\subsection{Experimentation with evaluation models}

Basically four models are used in organising development evaluations: the whole evaluation is out-sourced; the evaluation is split up into several individual external contracts; consultants are contracted to support an internal team leader; the evaluation is carried out by the evaluation unit.

Due to the complexity of the interactions between consultants and evaluation units during the evaluation processes and the lack of systematic knowledge about these processes, currently it is not possible analytically to define criteria, which can be applied in decisions on the organisation of the individual evaluations. As mentioned, 'independence' seems often to be a key argument in discussions on the organisation of evaluations, but the usual interpretation of 'independence' is very much linked to evaluation processes of more traditional interventions, for instance project assistance, without trying to understand how the characteristics of the evaluations can impact on the evaluation process and on both the actual and perceived independence of the evaluations. In addition, it may be argued that independence will not be considered equally important for all evaluations.

Therefore experience with different models should be collected more systematically. Already today there is a lot of variation, but monitoring is scarce, and the variation, to the extent it exists, can be found between donor agencies and rarely within agencies due to the fact that the individual agencies tend to use the same procedures for all their evaluations. Experiments within individual agencies with different organisational solutions for different types of evaluations and subsequent monitoring of these experiments would therefore be useful in order to develop practical criteria to be applied in decisions on organisational models.

Basic questions to explore are how different organisational solutions for the individual development evaluations influence, for example, the quality, credibility and use of the evaluations. A particular consideration will be whether there is a greater need for learning from some evaluations than others, and whether this should influence the choice of organisational model. For instance, a challenge is to conduct and learn from evaluations of complex interventions, which may require specific adapted organisational solutions. ${ }^{13}$

If evaluation processes should be adjusted to the characteristics of the individual evaluations several additional questions of a more institutional nature will have to be considered. First, who should decide on the organisation solution for the individual 
evaluation and will it be possible to develop some common norms and standards to guide this decision? Second, will donors be prepared to establish sufficient budget and institutional flexibility? For instance, the flexibility should comprise flexibility between budgets for consultants and budgets for in-house evaluation staff. Third, will a specific system for quality control that can ensure that common quality standards are applied for all evaluations - whatever organisational model used - have to be created? Today most development evaluations go through quality control before completion, but greater flexibility in the selection of organisational model may lead to calls for establishment of separate quality control mechanisms. Fourth, to which degree should the specific characteristics (for example, size of staff, budget and staff qualifications) of the individual evaluation units influence the selection of evaluation model?

\subsection{Experimentation with selection of consultants and TOR}

There are different models, which can be used in engaging consultants in evaluations, but most consultants are selected based on TOR and (a few) references. Although interviews are conducted it varies how much influence these interviews have on the final selection of the consultants. The basic requested qualifications of the evaluation consultants seem also to be quite similar from one evaluation to the next with a strong focus on the references from previous evaluations. In addition, a study found that many TORs do not detail the required competencies of the evaluation consultants (ITAD 2014). Thus, the selection process is basically the same whether it is a rather simple standard evaluation or a more complex evaluation.

The TORs for development evaluations have also been criticised for having too many questions and an insufficient description of the requested methodological approach. Some argue for the importance of having detailed and relatively specific TOR, even for complex evaluations (see Grävingholt and Leininger 2014: 168). However, desperate attempts to write very detailed TOR may not solve the problem as they may not reflect what is feasible in a specific evaluation, for example due to insufficient information at the time of drafting the TOR. Evaluations with many questions may instead lead to evaluations with a lack of depth (ITAD 2014: 72) and very general conclusions and recommendations (Andersen and Broegaard 2012). A potential implication can also be that not all questions in the TOR can be answered adequately. This is probably the reason for proposals of more openended and flexible TOR (Barnett and Bennett 2014: 55-6).

Another dimension will be the length of the assignment, where a complex evaluation may require a much longer time span in order to conduct an evaluation of reasonable quality.

An evaluation in Afghanistan showed the value of having given the evaluation consultants large flexibility and a longer time period to develop the methodology and complete the evaluation (Böhnke, Koehler and Zürcher 2014). A long-term contract is obviously close to an internal solution and may be met with the same questions related to quality control and independence, but can reduce the potential problem of insufficient internal evaluation capacity. In particular smaller evaluation units may not always be able to identify in-house evaluators with sufficient expertise within the areas to be evaluated. That there is an evaluation capacity problem has been noted in several analyses of donor agencies (see e.g. DFID 2014; ITAD 2014), and substantial training programmes have therefore been recommended.

As in the case of the two previous areas there is limited empirical knowledge about the significance of the selection procedures, the TORs, and of the contracts for different types of evaluations. Present information is based on a few analyses and a lot of anecdotal evidence, and apparently, in spite of quite a lot of normative work, there is no general agreement about how to deal with these elements in the evaluation process. Therefore, also in this area more empirical analyses are required.

\section{Concluding remarks}

As mentioned in the introduction, the discussions on development evaluation have in particular focused on methodological issues and on institutional solutions, which can ensure the independence of evaluations, while the evaluation processes have attracted less attention. The purpose of this article is to argue that the evaluation process plays a greater role than usually envisaged for the outcomes of development evaluations. 
In connection with the evaluation processes in particular four issues were identified. First, evaluation processes have generally not been revised to reflect the changing nature of development evaluation and upcoming new challenges, including a number of evaluations of complex interventions. Second, evaluation units tend to use the same 'system' for organising all their development evaluations in spite of significant variations in the characteristics of the evaluations, and other perspectives on the evaluation process than the 'independence perspective' have attracted less interest. Third, the evaluation process comprises a number of individual elements, which can be combined in different ways. These elements include, for example, consultant selection criteria, TOR, types of contracts, different kinds of quality control, etc. Fourth, only few empirical analyses of the evaluation processes are available with the implication that systematic empirical knowledge about the evaluation process and its elements is limited. These four issues together raise the important question as to whether - due to a concern for evaluation independence - evaluation processes are organised in a way which may not lead to development evaluations of the desired quality.

In order to establish a better basis for taking decisions on the organisation of evaluations, it

\section{Notes}

* I would like to thank Barbara Befani, Eva Broegaard and Megan Kennedy-Chouane for a number of excellent comments to a previous draft of this article. The views expressed in the article are those of the author and do not necessarily represent the views of the Ministry of Foreign Affairs of Denmark.

1 See for example White (2009) and Stern et al. (2012) for different perspectives on this discussion and with a particular focus on impact evaluations.

2 See for example Ling (2012) and Picciotto (2012) for introductions to recent discussions, including to the distinction between simple, complicated and complex interventions.

3 Many stakeholders, including staff from donor evaluation units, seem to agree - at least informally - that there is a problem with the quality of many development evaluations (see for example Picciotto 2011: 171 and 2012: 220, which explicitly mention the low quality of many development evaluations. See also ITAD 2014). was suggested, using the political economy perspective as an analytical framework, to conduct empirical analyses of the incentives and strategies of evaluation consultants and evaluation units in connection with different kinds of evaluations. Further, it was proposed to monitor experiments with different evaluation models and their elements, such as consultant requirements, TOR, contract types and quality control, and to assess the implication on evaluation quality, credibility and use.

The evaluation process is just one contributing factor to the final outcome of an evaluation, but as the way the evaluation process is designed has implications for the incentives of the various actors, the evaluation process should reflect the purpose and characteristics of the individual evaluations. Many consultants and evaluation units are aware of this, but practical reasons and formal restrictions may prevent them from adjusting the way evaluation processes are managed.

The indicated empirical analyses and experiments may be met with some resistance due to vested interests and will be difficult to conduct methodologically, but may also provide important new insights in the development evaluation processes and facilitate future improvements in development evaluation quality.

4 See for example Eliadis, Furubo and Jacob (2011: 55), which mentions that the 'methodological and scientific dimensions... overshadow the power dimension and does not allow for a full grasp of the issues, which drive the evaluation process' and Picciotto (2013: 18), which mentions that 'the governance dimension of evaluation is rarely addressed'.

5 In this article 'consultant' and 'evaluation consultant' comprise those who are contracted to conduct development evaluations, including consultancy companies, freelance consultants, research institutes, universities, etc.

6 www.oecd.org (accessed 26 June 2014).

7 It should be noted that this estimate is only for development evaluations. Development reviews, appraisals, etc. are also conducted by development consultants, and it can be assumed that the budgets for these tasks are much higher.

8 Due to lack of data it is not possible to provide information on the specific composition of different types of contracts (short-term, longterm, framework contracts, etc.). 
9 Note that Picciotto (2011) makes a distinction between models based on a competitive market and professionalism. This distinction will not be made in this article.

10 For introductions to transaction cost economics, see the work of Oliver Williamson (e.g. Williamson 1998). There are only a few attempts to operationalise the transaction cost perspective in connection with development evaluation (see e.g. Jobin 2008 and Andersen and Broegaard 2012).

\section{References}

Andersen, O.W. and Broegaard, E. (2012) 'The Political Economy of Joint Donor Evaluations', Evaluation 18.1: 47-59

Barnett, C. and Bennett, J. (2014) 'Critical Reflections on the South Sudan Evaluation of Conflict Prevention and Peacebuilding Activities', in O.W. Andersen, B. Bull and M. Kennedy-Chouane (eds), Evaluation Methodologies for Aid in Conflict, London and New York NY: Routledge: 38-60

Böhnke, J.; Koehler, J. and Zürcher, C. (2014) 'Assessing Development Cooperation in Northeast Afghanistan with Repeated Mixedmethods Surveys', in O.W. Andersen, B. Bull and M. Kennedy-Chouane (eds), Evaluation Methodologies for Aid in Conflict, London and New York NY: Routledge

DFID (2014) Rapid Review of Embedding Evaluation in UK Department for International Development, Final Report, London: Department for International Development

Eliadis, P.; Furubo, J.-E. and Jacob, S. (eds) (2011) Evaluation: Seeking Truth or Power?, Comparative Policy Evaluation 17, New Brunswick NJ and London: Transaction Publishers

Furubo, J.-E. and Vestman, O.K. (2011) 'Evaluation: For Public Good or Professional Power', in P. Eliadis, J.-E. Furubo and S. Jacob (eds), Evaluation: Seeking Truth or Power? Comparative Policy Evaluation 17, New Brunswick NY and London: Transaction Publishers

Grävingholt, J. and Leininger, J. (2014) 'Evaluating Statebuilding Support: Learning from Experience or Judging from Assumptions?', in O.W Andersen, B. Bull and M. KennedyChouane (eds), Evaluation Methodologies for Aid in Conflict, London and New York NY: Routledge: 154-74

ITAD in association with Chr. Michelsen Institute (2014) Can We Demonstrate the
11 See for example Andersen and Broegaard (2012), where further references can be found.

12 Apparently, there is an increasing interest for conducting analyses within this area, but usually without distinguishing between different kinds of development evaluations (see e.g. DFID 2014; ITAD 2014).

13 Embedded evaluations have been seen as a solution to this learning objective, but as shown in a recent review, embedding evaluations may lead to particular challenges (DFID 2014).

Difference that Norwegian Aid Makes? Evaluation of Results Measurement and How This Can Be Improved, Report 1/2014, Oslo: NORAD Jacob, S. and Boisvert, Y. (2010) 'To Be or Not to Be a Profession: Pros, Cons and Challenges for Evaluation', Evaluation 16.4: 349-69

Jobin, D. (2008) 'A Transaction Cost-based Approach to Partnership Performance Evaluation', Evaluation 14.4: 437-65

Ling, T. (2012) 'Evaluating Complex and Unfolding Events in Real Time', Evaluation 18.1: 79-81

OECD (2010a) Evaluation in Development Agencies, Better Aid, Paris: OECD Publishing

OECD (2010b) DAC Evaluation Quality Standards. DAC Evaluation Network, Paris: OECD Publishing

Picciotto, R. (2013) 'Evaluation Independence in Organizations', Journal of MultiDisciplinary Evaluation 9.20: 18-32

Picciotto, R. (2012) 'Experimentalism and Development Evaluation: Will the Bubble Burst?', Evaluation 18: 213-29

Picciotto, R. (2011) 'The Logic of Evaluation Professionalism', Evaluation 17.2: 165-80

Stern, E.; Stame, N.; Mayne, J.; Forss, K.; Davies, R. and Befani, B. (2012) Developing a Broader Range of Rigorous Designs and Methods for Impact Evaluations, report of a study commissioned by the Department for International Development (DFID), London: DFID

United Nations (2013) A New Global Partnership: Eradicate Poverty and Transform Economies Through Sustainable Development, New York NY: United Nations Publications

White, H. (2009) 'Theory-based Impact Evaluation: Principles and Practice', Journal of Development Effectiveness 1.3: 271-84

Williamson, O. (1998) 'Transaction Cost Economics: How it Works; Where it is Headed', De Economist 146: 23-58 